\title{
Ultra-sensitive silicon nitride waveguide-enhanced Raman spectroscopy for aqueous solutions of organic compounds
}

\author{
Zuyang Liu ${ }^{1,2 *}$, Haolan Zhao ${ }^{1,2}$, Bettina Baumgartner ${ }^{3}$, Bernhard Lendl ${ }^{3}$, Andre \\ Skirtach $^{2,4}$, Nicolas Le Thomas ${ }^{1,2}$, Roel Baets ${ }^{1,2}$ \\ ${ }^{1}$ Photonics Research Group, Ghent University-IMEC, 9052 Ghent, Belgium \\ ${ }^{2}$ Center for Nano- and Biophotonics, Ghent University, 9052 Ghent, Belgium \\ ${ }^{3}$ Institute of Chemical Technologies and Analytics, Technische Universität Wien, 1060 Vienna, Austria \\ ${ }^{4}$ Department of Biotechnology, Ghent University, 9000 Ghent, Belgium \\ Zuyang.Liu@UGent.be
}

\begin{abstract}
We demonstrate a waveguide-enhanced Raman sensor functionalized with mesoporous silica coating for organic compounds in aqueous solutions. The detection limit of cyclohexanone in water is improved by at least 100 times compared to bare waveguides.

(C) 2020 The Author(s)
\end{abstract}

For accurate identification and rapid quantification of one or more compounds simultaneously, Raman spectroscopy is a promising candidate, as it probes the characteristic vibrations of molecules in the fingerprint region of the spectrum. A major challenge intrinsic to Raman spectroscopy is the extremely weak signal. To circumvent this limitation, one method is waveguide-enhanced Raman spectroscopy, which excites and collects the Raman signal of an analyte via a photonic waveguide. The tight confinement of the optical field and the long interaction length enhance the light-matter interaction [1]. Furthermore, the chip-based approach holds the promise of very compact and cost-effective Raman sensors. Silicon nitride $\left(\mathrm{Si}_{3} \mathrm{~N}_{4}\right)$ waveguides have been widely explored for waveguide-enhanced Raman spectroscopy for various analytes, including bulk liquids [1] and biological monolayers [2]. With the assistance of functionalized coatings, sensing of trace gas and volatile organic compounds has been realized by utilizing $\mathrm{Si}_{3} \mathrm{~N}_{4}$ waveguides $[3,4]$.

Mesoporous silica has often been used as an excellent adsorbent of various categories of compounds in diverse applications, such as catalysis [5] and sensors [6]. Depending on the functionalization of mesoporous silica, different target molecules can be adsorbed. For instance, a mesoporous silica film with its surface modified by hexamethyldisilazane (HMDS) shows enrichment of non-polar compounds in aqueous solutions. This type of mesoporous silica holds strong potential in monitoring organic compounds in aqueous solutions at low concentrations, as the organic molecules usually exhibit low polarity.

In this work, we demonstrate a waveguide-enhanced Raman sensor for low concentration organic compounds in aqueous solutions, realized with $\mathrm{Si}_{3} \mathrm{~N}_{4}$ slot waveguides and mesoporous silica coatings functionalized by HMDS. The $\mathrm{Si}_{3} \mathrm{~N}_{4}$ slot waveguide has two $300 \mathrm{~nm}$ wide rails separated by a gap with a width of $150 \mathrm{~nm}$, and the waveguide length is approximately $1 \mathrm{~cm}$. As sketched in Fig. 1, the waveguide is coated with a thin mesoporous silica film that fills the slot. The coating was modified by HMDS, as described in [7]. In our experiments, we used a $785 \mathrm{~nm}$ pump laser to excite the fundamental quasi-TE mode and the Raman signal is analyzed by a spectrometer (equipped with Andor iDus 401 camera). The objective used for coupling light into and out from the waveguide has a 40x magnification and an NA of 0.6. The pump power before objective is $60 \mathrm{~mW}$. The integration time of all measurements is $0.05 \mathrm{~s}$. The analyte we chose for the experiment is cyclohexanone, a hydrophobic compound that is commonly used as a solvent in industry.

We first measure the Raman response of DI water with the functionalized waveguide. Next, aqueous solutions of cyclohexanone with different volume concentrations $(0.01 \%-1 \%)$ were applied onto the waveguide successively. The sample was rinsed with isopropyl alcohol (IPA) between measurements, which allows to remove the adsorbed cyclohexanone molecules. As a comparison, we have measured the Raman signal from cyclohexanone from a bare waveguide, and the result is shown in Fig. 2. As illustrated in Fig.2(a), the red curve is the Raman spectrum of $1 \%$ cyclohexanone in DI water with its background subtracted, which shows an outstanding correspondence with a reference cyclohexanone Raman spectrum from [8]. The strongest peak around $750 \mathrm{~cm}^{-1}$ is then fitted with a Lorentzian function to obtain its area under the peak (blue line). Fig.2(b) shows the fitted area under the peak for different concentrations of cyclohexanone solutions, with the error bar representing the fitting error. We then fitted the adsorption isotherm with the Freundlich model [9], of which the coefficients are listed in Fig.2(b). A significant enrichment of cyclohexanone can be seen from the comparison between coated and bare $\mathrm{Si}_{3} \mathrm{~N}_{4}$ slot waveguides. For a bare $\mathrm{Si}_{3} \mathrm{~N}_{4}$ slot waveguide, the signal is hardly resolvable at $1 \%$ cyclohexanone, while the coated waveguide 
(a)

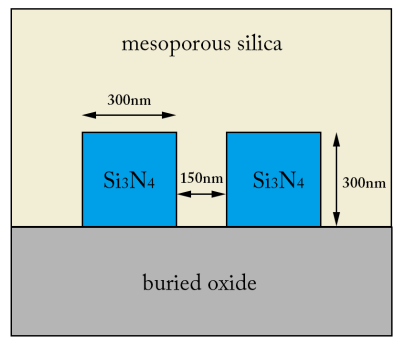

(b)

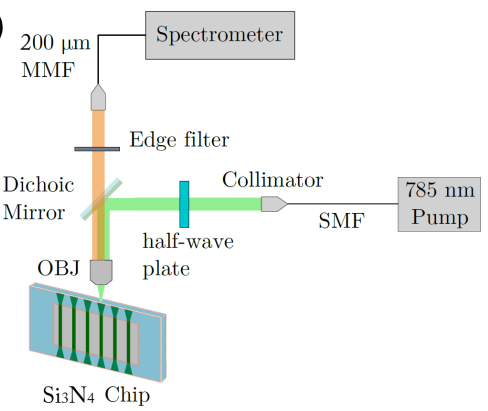

Fig. 1. (a) Cross-section of the functionalized $\mathrm{Si}_{3} \mathrm{~N}_{4}$ slot waveguide. (b) Experiment setup.

generated obvious signal at $0.01 \%$. Therefore, the detection limit of cyclohexanone in water is improved by at least 100 times. From the fitted Freundlich model of the coated waveguide - and taking into account noise - we estimate that the detection limit can be improved by 10000 times. The dramatic improvement is mainly due to the strongly nonlinear nature of the Freundlich curve.
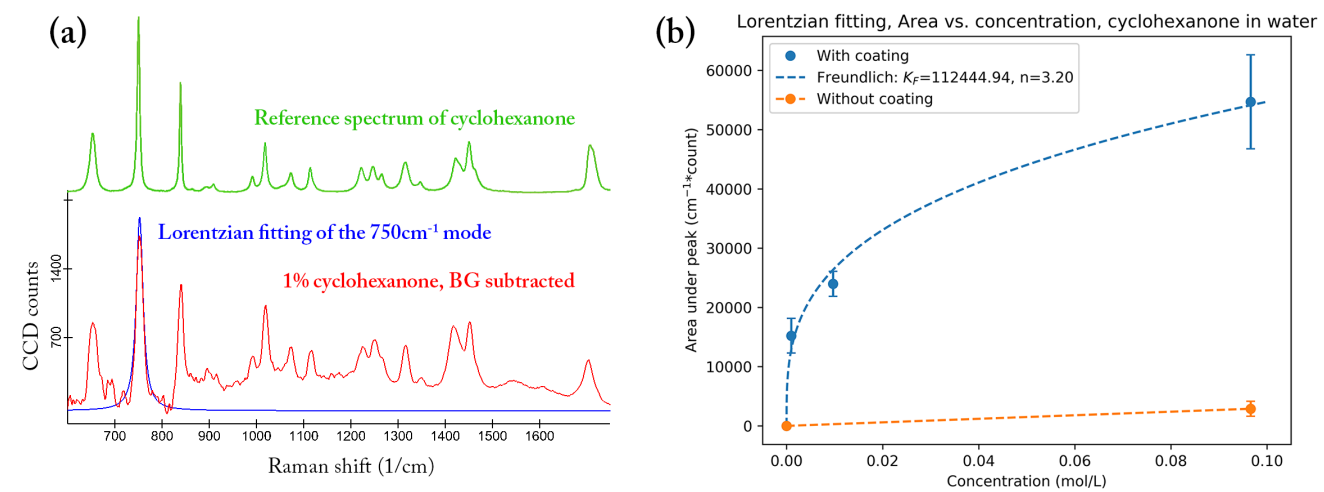

Fig. 2. (a) Red curve: Raman spectrum of $1 \%$ cyclohexanone after subtraction of Raman background. Blue curce: Lorentzian fitting of the peak around $750 \mathrm{~cm}^{-1}$. Green curve: reference spectrum of cyclohexanone [8]. (b) Fitted area under peak of different applied concentrations of cyclohexanone, on coated and bare $\mathrm{Si}_{3} \mathrm{~N}_{4}$ slot waveguides. The result of coated waveguide is fitted with Freundlich model of adsorption.

In conclusion, by utilizing mesoporous silica for waveguide-enhanced Raman spectroscopy, we improved the detection limit of cyclohexanone in aqueous solution by at least 100 times (demonstrated), and potentially 10000 times (estimated). This ultra-sensitive Raman sensor brings us one step closer to the monitoring of organic compounds in aqueous solutions in industrially and environmentally relevant concentrations.

\section{References}

1. A. Dhakal, A. Z. Subramanian, P. Wuytens, F. Peyskens, N. Le Thomas, and R. Baets, Opt. Lett. 39, 40254028 (2014).

2. A. Dhakal, P. C. Wuytens, F. Peyskens, K. Jans, N. Le Thomas, and R. Baets, ACS Photonics 3, 2141-2149 (2016).

3. S. Holmstrom, T. Stievater, D. Kozak, M. Pruessner, N. Tyndall, W. Rabinovich, R. McGill, and J. Khurgin, Optica 3, 891-896 (2016).

4. H. Zhao, A. Raza, B. Baumgartner, S. Clemmen, B. Lendl, A. Skirtach, and R. Baets, in Conference on Lasers and Electro-Optics, OSA Technical Digest (Optical Society of America, 2019), paper STh1F.7.

5. J. H. Clark, D. J. Macquarrie, and S. Tavener, Dalt. Trans. 0, 4297-4309 (2006).

6. B. J. Melde, B. J. Johnson, and P. T. Charles, Sensors 8, 5202-5228 (2008).

7. B. Baumgartner, J. Hayden, A. Schwaighofer, and B. Lendl, ACS Appl. Nano Mater. 1, 7083-7091 (2018).

8. Bio-Rad Laboratories, Inc. SpectraBase (2019)

9. Y. S. Ho, Carbon 42, 2115-2116 (2004). 